\title{
OULIPO: UN VIAJE DESDE LAS MATEMÁTICAS A LA LITERATURA
}

\author{
Marta MACHO STADLER \\ Universidad del País Vasco - Euskal Herriko Unibertsitatea \\ marta.macho@ehu.es
}

Tome una palabra tome dos póngalas a cocinar como si fuesen huevos tome una pizca de sentido luego un gran trozo de inocencia caliente a fuego lento al fuego lento de la técnica vierta la salsa enigmática sazone con algunas estrellas

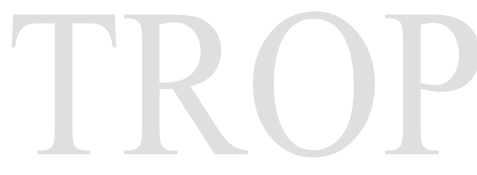
eche pimienta y luego arríe las velas ¿Adónde quiere llegar? A escribir ¿Realmente? ¿¿A escribir??

\section{1. ¿Qué es OuLiPo?}

Raymond Queneau, Pour un art poétique ${ }^{1}$

OuLiPo - Ouvroir de Littérature Potentielle ${ }^{2}$ - se creó en noviembre de 1960 a iniciativa de Raymond Queneau —un hombre de letras con gusto por las matemáticas- y François Le Lionnais ${ }^{3}$ - un hombre de ciencias, con gusto por la literatura-, y secundados por un grupo de escritores, matemáticos y pintores.

Raymond Queneau explicaba de esta manera su visión de OuLiPo:

¿Qué es la literatura potencial? Yo diría en primer lugar que es a lo que se dedica el grupo fundado hace tres años por François le Lionnais. Consta de diez miembros y ha tomado el nombre de Ouvroir de Littérature Potentielle:

Ouvroir porque pretende obrar

Littérature porque se trata de literatura

Potentielle, la palabra debe tomarse en distintos sentidos que aparecerán, espero, a lo largo de esta exposición

En resumen: OU.LI.PO

¿Cuál es el objetivo de nuestros trabajos? Proponer a los escritores nuevas «estructuras», de naturaleza matemática o aun inventar nuevos procedimientos artificiales o mecánicos, para contribuir a la actividad literaria: apoyos a la inspiración, por así decirlo, o aun, de alguna manera, una ayuda a la creatividad.

\footnotetext{
${ }^{1}$ Traducido por la autora (Queneau, 1965a).

${ }^{2}$ Obrador de Literatura Potencial, www.oulipo.net

${ }^{3}$ Es el «Frésident-Pondateur».
} 
¿Qué es lo que no es el OU.LI.PO?

1. No es un movimiento o una escuela literaria. Nos ponemos por debajo del valor estético, lo que no significa que lo ignoremos.

2. No es tampoco un seminario científico, un grupo de trabajo «serio» entre comillas, aunque formen parte de él un profesor de la Facultad de Letras y un profesor de la Facultad de Ciencias. [...]

3. No se trata de literatura experimental o aleatoria [...].

Diré ahora lo que es -o más bien lo que cree ser- OU.LI.PO. Nuestras investigaciones son:

1. ingenuas: tomo la palabra «ingenua» en su sentido perimatemático, del mismo modo que se dice teoría «ingenua de conjuntos». Avanzamos sin refinar demasiado. Intentamos demostrar el movimiento andando.

2. artesanales - aunque esto no es lo esencial一. Nos afligimos por no disponer de máquinas: lamento continuo en todas nuestras reuniones.

3. divertidas: al menos para nosotros. Algunos las encuentran «un aburrimiento sórdido», lo que no debería asustarnos, porque no estáis aquí para divertiros. [...]

Una parte de nuestra actividad es de orden histórico, es decir, consiste en buscar lo que ha podido hacerse de análogo en el pasado. Es una tarea inmensa (Queneau, 1965b; traducción de la autora).

Por su parte, François Le Lionnais expresaba en estos términos el método de trabajo al que aspiraba OuLiPo:

Es posible componer textos que tendrán cualidades poéticas, surrealistas, fantásticas u otras, sin tener cualidades potenciales. Pero es este último rasgo el que es esencial para nosotros. Es el único que debe guiar nuestra elección... El fin de la literatura potencial es proveer a los escritores futuros de técnicas nuevas que puedan reservar la inspiración de su afectividad. De allí la necesidad de una cierta libertad. Hace nueve o diez siglos, cuando un literato potencial propuso la forma del soneto dejó, a través de ciertos procedimientos mecánicos, la posibilidad de una elección. [...]

Así, hay dos lipos: una analítica y una sintética. La lipo analítica busca posibilidades que se encuentran en ciertos autores, sin que ellos lo hubieran pensado. La lipo sintética constituye la gran misión de OuLiPo: se trata de abrir nuevas posibilidades desconocidas para los autores antiguos. [...]

El OuLiPo tiene como objetivo descubrir estructuras nuevas y de dar para cada una de ellas ejemplos en pequeña cantidad (Oulipo, 1973; traducción de la autora).

Para sus propósitos, OuLiPo se concentró en dos tareas:

1. la invención de nuevas estructuras y retos — la lipo sintética - mediante la combinación de literatura y matemáticas,

2. el examen de obras literarias antiguas ${ }^{4}$ - la lipo analítica - con el objetivo de encontrar rastros del uso de estructuras, formas o restricciones.

OuLiPo rechaza la inspiración como única fuente de creatividad — se desmitifica el trabajo literario, convirtiéndolo en una tarea artesanal-: la restricción es el motor creativo.

Tras los primeros años de trabajo, se han ido creando diferentes OuXPo —obrador de ' $\mathbf{X}$ ' potencial—: OuLiPoPo - X=literatura policial - en 1973; OuCiPo $-\mathbf{X}=$ cine - en 1974, fundado por el grupo danés DOGMA 95 siguiendo un estricto manifiesto de Lars von Trier, Tomas Vinterberg y Soren Kragh-Jacobsen; OuPeinPo $-\mathbf{X}=$ pintura - en 1981; OuTraPo $-\mathbf{X}=$ teatro- en 1991; OuBaPo $-\mathbf{X}=$ tebeo- en 1992, etc.

\footnotetext{
${ }^{4}$ A los autores de estas obras los denominaban «plagiarios por anticipación»: François Le Lionnais, Le Second manifeste (Oulipo, 1987b).
} 
OuLiPo consta de treinta y ocho miembros ${ }^{5}$ de todas las edades, profesiones, nacionalidades, y, como comenta Marcel Bénabou ${ }^{6}$, les une «el interés por la escritura bajo restricción, el gusto por compartir y relacionarse, una cierta forma de humor...» (Bénabou, 2001; traducción de la autora).

No es preciso pertenecer al «selecto grupo» para hacer literatura «oulipiana», y tampoco todo lo que los miembros de OuLiPo producen puede calificarse como «literatura potencial».

Las $_{\text {contraintes }}{ }^{7}$-constricciones, trabas, restricciones- que se imponen a la hora de crear sus textos están en continua evolución: los miembros de OuLiPo proponen nuevas trabas continuamente. Además, un buen autor oulipiano debe explicar la restricción que está utilizando en el texto que presenta.

En mi caso, mi fascinación por la literatura oulipiana se debe fundamentalmente al juego encerrado en sus propuestas: el desafío contenido en sus trabas, la fina ironía y la inclusión de las matemáticas de diferentes maneras, forman — desde mi punto de vista - un complemento notable a las letras de estos autores.

En los siguientes apartados explicaré algunas trabas oulipianas a través de mi mirada matemática; sin duda, las elecciones de los textos se han basado tan sólo en mis gustos como lectora.

\section{Los Fundamentos de la literatura según David Hilbert}

Queneau transformó los Grundlagen der Geometrie de 1899 de David Hilbert, en los Fondements de la littérature d'après David Hilbert. En este texto presenta una axiomática de la literatura, reemplazando en las proposiciones originales de Hilbert las palabras «punto», «recta»y «plano», por «palabra», «frase», «párrafo», respectivamente. El resultado es sorprendente —a veces se trata de pura lógica y, en otras ocasiones, el axioma parece un tanto extraño-, un divertido juego

\footnotetext{
${ }^{5}$ Son todas las personas que han formado parte del grupo en algún momento. No se deja de pertenecer a OuLiPo sólo por haber fallecido, aunque el escritor Eduardo Berti me apuntó hace unas semanas que el suicidio jes causa de expulsión inmediata!

${ }^{6}$ Marcel Bénamou es el «Secrétaire définitivement provisoire» de OuLiPo desde 1970.

${ }^{7}$ Extraídas de la página web de OuLiPo: «À suppposer..., Abécédaire, Acronyme, Acrostiche brivadois, Acrostiche universel, Alexandrin greffé, Alexandrin jouetien, Alexandrin oral, Algorithme de Mathews, Alva, Anaérobie, Anagramme, Antérime, Antirime, Aphorime, Aphorisme, Arbre à théâtre, Arbres et arborescence, Avalanche, Avion, Baobab, Beau présent, Belle absente, Bibliothèques virtuelles, Bord de poème, Boule de neige, Bris de mots, Bubu l'Urubu, Caradec, Carré lescurien, Chicago, Chimère, Chronopoème, Citations, CMMP, Conte à votre façon, Contrainte de Delmas, Contrainte de Lloyd, Contrainte de Pascal, Contrainte de Turing, Contrainte du prisonnier, Cornichon, Cylindre, Désarguesienne, Deunglitsch, Echelle, Eclipse, Emir, Eodermdrome, Epithalame oulipien, Erim, Etreinte, Exercice de style, Explorations à la limite, Filigrane, Formes fixes, Graphe, Haï-kaïsation, Hétérogrammes, Homomorphisme, Homophonies, Homosyntaxisme, Homovocalisme, Hypertropes, Immorale élémentaire, Intérieur de poème, Inventaire, Joséphine, Leiris, Lipogramme, Liponymie, Lipossible, Littérature définitionnelle, Locurime, Locutions introuvables, Logo-rallye, LSD, L'égal franglais, Minisextine, Mongine, Monkine, Monoconsonnantisme, Monostiche paysager, Monovocalisme, Morale élémentaire, N-ine, Oblique, Onzain hétérogrammatique, Ouliporime, Palindrome, Parcours obligé (ou logo-rallye), Perverbe, Petite boîte, Petite morale élémentaire portative, Poème carré ou carré lescurien, Poème de métro, Poème fondu, Poème pour bègue, QSSD, Quenine, Quenine à démarreur, Queninisation, Quenoum, Redonde, Rime berrichonne, Rime bisexuelle, Rime de début, S+7, Sardinosaure, Sollicitude, Sonnet à la limite, Sonnet irrationnel, Sonnet mince, Surdéfinitions, Terine, Terine à triolet, Terine aux trois voyelles, Terine syllabique, Textes à démarreur, Théâtre booléen, Tireur à la ligne, Traduction antonymique, Traduction homophonique, Transduction, Trident, Ulcérations, V+7, Variation sur S+7, Villanelle, Vocabulaires raisonnés, X prend Y pour Z, etc.».
} 
en el que se observa que la axiomática de la geometría plana no se traslada con facilidad al mundo de la literatura:

I. 1. Existe una frase conteniendo dos palabras dadas. [...]

I. 2. No existe más que una frase conteniendo dos palabras dadas. [...]

I. 3. En una frase hay al menos dos palabras; existen al menos tres palabras que no pertenecen a la misma frase. $[\ldots]$

I. 4a. Existe un párrafo que contiene tres palabras que no pertenecen a la misma frase. [...]

I. 4 b. Todo párrafo contiene al menos una palabra. [...]

I. 5. No existe más de un párrafo conteniendo tres palabras que no pertenecen todas a la misma frase. $[\ldots]$

I. 6. Si dos palabras de una frase pertenecen a un párrafo, todas las palabras de esta frase pertenecen a este párrafo. $[\ldots]$

I. 7. Si dos párrafos tienen una palabra en común, tienen también otra en común. [...]

I. 8. Existen al menos cuatro palabras que no pertenecen al mismo párrafo. [...]

Teorema 1 (Hilbert): Dos frases distintas de un mismo párrafo tienen a lo más una palabra en común; dos párrafos distintos o bien no tienen ninguna palabra en común o bien tienen en común una frase y no tienen ninguna palabra en común fuera de esta frase. [...]

II. 1. Si en una frase una palabra se encuentra entre dos palabras tomadas en un orden dado, también se encuentra entre estas dos palabras tomadas en orden inverso. [...]

II. 2. Dadas dos palabras de una frase, existe al menos una tercera palabra, tal que la segunda esté entre la primera y la tercera. [...]

II. 3. De tres palabras de una frase, hay una que se encuentra entre las otras dos. [...]

II. 4. Sean tres palabras de un párrafo no pertenecientes todas a la misma frase y sea una frase no conteniendo estas tres palabras, pero del mismo párrafo. Si esta frase contiene una palabra de una frase determinada por dos de estas palabras, contendrá siempre una palabra común con la frase determinada por una de estas palabras y la tercera. [...]

Teorema 3: Dadas dos palabras, la frase donde figuran contiene al menos una palabra entre estas dos. $[\ldots]$

Teorema 7: Entre dos palabras de una frase existe una infinidad de ellas (Oulipo, 1987a; traducción de la autora).

Para intentar aclarar esta axiomática tan excéntrica, Queneau explica más adelante:

Para controlar esta sorpresa y comprender estos teoremas, hay que admitir simplemente la existencia de, siguiendo el ejemplo de la vieja geometría proyectiva, lo que llamaríamos «palabras imaginarias» y «palabras en el infinito». Toda frase contiene una infinidad de palabras; sólo se aprecia un número muy limitado, las demás se encuentran en el infinito o son imaginarias. Muchas mentes habían tenido este presentimiento, pero nunca la conciencia neta. Es imposible para la retórica no tener ya en cuenta este teorema capital. La lingüística podrá igualmente sacar provecho.

¡A mí me parece sublime! 


\section{3.- Sextinas: combinatoria y poesía}

Una sextina ${ }^{8}$ es un poema formado por seis estrofas de seis versos cada una de ellas, que finaliza con una contera de tres versos. Sólo hay seis palabras que generan la rima y se distribuyen de acuerdo con el siguiente esquema ${ }^{9}$ :

\section{ABCDEF - FAEBDC - CFDABE - ECBFAD - DEACFB - BDFECA - ECA}

En el anterior diseño se está describiendo lo que en matemáticas se denomina una permutación — se permutan las seis palabras al cambiar de estrofa—; pero se trata además de una permutación de orden 6 , es decir, cuando se hacen seis iteraciones - y no antes- se reencuentran las palabras de rima en su forma original. Si llamamos $\sigma$ a esta permutación, se escribe del modo

$$
\left(\begin{array}{llllll}
1 & 2 & 3 & 4 & 5 & 6 \\
2 & 4 & 6 & 5 & 3 & 1
\end{array}\right)
$$

Y es $\sigma^{6}=\mathrm{id}^{10}$, pero $\sigma \neq \mathrm{id}, \sigma^{2} \neq \mathrm{id}, \sigma^{3} \neq \mathrm{id}, \sigma^{4} \neq \mathrm{id}$ y $\sigma^{5} \neq \mathrm{id}$.

Para constatar la complejidad de esta forma poética, se transcribe debajo la sextina Rabia y miel de Sofía Rhei. Su estructura no es la misma que la de Arnaut Daniel ${ }^{11}$, pero sigue siendo una permutación de orden 6 ,

$$
\left(\begin{array}{llllll}
1 & 2 & 3 & 4 & 5 & 6 \\
2 & 4 & 5 & 3 & 6 & 1
\end{array}\right)
$$

es decir, cada palabra pasa una vez y sólo una por cada una de las seis líneas de cada estrofa:

\section{ABCDEF - FADBCE - EFBADC - CEAFBD - DCFEAB - BDECFA}

La prima canta, albina, abierta al aire desde el brillante borde, desde el cobre perforado en matriz para la sangre de la guitarra, en su redonda llama de combustión sonora. Pinza, araña el meñique, y estalla cada golpe.

Es la alegría en la vértebra un golpe de agudo evaporado, hecho del aire, un arpegio de puro nervio y llama que se respira, es la alegría cobre que destella, caricias en la sangre: cada palma que ríe es una araña,

y entre palos, araña y araña se encuentran en el más preciso golpe. Pero no nace el verdadero cobre del ritmo en otro sitio que en el aire donde ojos y bocas prenden llama, prenden compás al tiempo de la sangre.

\footnotetext{
${ }^{8}$ El trovador provenzal Arnaut Daniel fue el creador de esta forma poética; la primera sextina de la historia de la literatura es su Lo ferm voler qu'el cor m'intra.

${ }^{9}$ En la mayor parte de los casos, vuelven a aparecer las seis palabras en la contera final.

${ }^{10} i d$ es la identidad, la configuración original.

${ }^{11}$ La sextina de Arnaut Daniel tiene además una estructura en espiral (Audin, 2010).
} 
Siempre algo más necesita la sangre, algo como la rabia que arde, araña y se derrama en el cálido aire de la sangre como un acorde; el golpe de un vértigo de miel, de oro, de cobre cálido y dulce, de líquida llama.

No existe luz que no ciegue, no hay llama que no haga crepitar hasta la sangre, no hay música si no hay también el golpe. La rabia que es la miel, la miel que araña es sal, de rabia y gritos en el aire: se está secando de la piel el cobre.

Tiembla el pulgar, tan flaco como el cobre de la cuerda más grave. Ya no hay llama en el mirar, porque la negra araña de párpados que inflama espesa sangre no deja ver los ojos. Se oye el golpe con el que sale del pulmón el aire.

Se va el callado tiempo con que el aire araña hasta la sangre en una llama de cobre.

Con un golpe,

el bordón llora

(Arellano-Munárriz-Rhei, 2011; publicado con permiso de la autora).

Como se observa, Sofía Rhei se permite además una licencia en la contera final al añadirle dos versos adicionales.

Raymond Queneau (Oulipo, 2003) ${ }^{12}$ se preguntó si era posible generalizar la estructura de la sextina, reemplazando 6 por $n$, para escribir un poema de $n$ estrofas, cada una formada por $n$ versos, todos terminados por las mismas $n$ palabras, permutadas por la aplicación:

$$
\sigma(p)=\left\{\begin{array}{rll}
2 p & \text { si } & p \leq \frac{n}{2} \\
2(n-p)+1 & \text { si } & p>\frac{n}{2}
\end{array}\right.
$$

En su honor, este tipo de poema pasó a denominarse una quenina de orden n. No existen queninas de cualquier orden, debido a una incompatibilidad matemática. Por ejemplo, no existen las de orden 4 , porque con la fórmula propuesta por Queneau, es $\sigma(1)=2, \sigma(2)=4, \sigma(3)=3$ y $\sigma(4)=1$, y se comprueba con facilidad que $\sigma^{3}=\mathrm{id}$. Tampoco existen 10-queninas: en este caso la permutación es de orden 7 , no de orden 10.

Para las queninas de orden 11 —éstas existen- Georges Perec da una respuesta al reto de Queneau:

Cada uno de los ciento setenta y seis textos de este libro es una oncena, un poema de once versos, en el que cada verso tiene once letras. Cada verso usa una misma serie de letras diferentes, una especie de gama, cuyas permutaciones producirán un poema según un principio análogo al de la música serial: no se puede repetir una letra antes de haber agotado la serie. Todos los poemas tienen en común las diez letras más frecuentes del alfabeto francés E, S, A, R, T, I, N, U, L, O. La undécima letra es una de las dieciséis restantes

\footnotetext{
${ }^{12}$ Jacques Roubaud, N-ine, autrement dit quenine (encore).
} 
B, C, D, F, G, H, J, K, M, P, Q, V, W, X, Y, Z. Hay once poemas en B, once en C, etc., en total once alfabetos completos, es decir $16 \times 11=176$ poemas.

La disposición tipográfica de los textos hace visible esta traba, dando a cada poema dos disposiciones diferentes: una de ellas está ordenada en un cuadrado de $11 \times 11$ y la otra es libre y propone una especie de traducción en prosa de este poema (Perec, 1976; traducción de la autora).

De hecho puede probarse $^{13}$ que:

Si existe una quenina de orden $n$ (con la fórmula dada por Queneau), entonces $2 n+1$ es un número primo $^{14}$. Además, si $2 n+1$ es primo, existe una quenina de orden $n$ si y sólo si 2 es de orden $2 n$ o de orden $n$ módulo $2 n+1^{15}$.

Otra variante de sextina es la propuesta por Jacques Roubaud (Roubaud, 1985, 1987 y 1990) en las aventuras de Hortense. La primera idea del autor era la de elaborar una sextina de sextinas -en prosa-, con seis novelas subdivididas cada una de ellas en seis partes, divididas a su vez en seis capítulos. La serie quedó finalmente reducida a tres novelas, pero la alusión a esta forma de poesía se deja ver en el texto continuamente. Por ejemplo, cuando el autor habla del orden de sucesión de los príncipes herederos en Poldavia:

El orden de prelación entre los Príncipes se modificaba en cada generación, siguiendo una permutación fijada inmutablemente desde el siglo XIII, cuando los príncipes de entonces habían puesto fin a sus sanguinarias querellas: el hijo mayor del primer Príncipe Reinante se transformaba en el segundo en el orden jerárquico (podía ser la hija mayor y se convertía entonces en Princesa Reinante número 2), el heredero (o heredera) del segundo cuarto, el tercero pasaba a la sexta posición, el cuarto a la quinta y el quinto a la tercera; en cuanto al sucesor del Sexto Príncipe (chica o chico), se transformaba en primero; de esta manera, como percibe usted fácilmente con un pequeño cálculo, querido Lector, cada familia ocupaba sucesivamente cada lugar en la jerarquía. El orden inicial, el del Primer Príncipe (Arnaut Danieldzoï ${ }^{16}$ ), quedaba restablecido al cabo de seis generaciones y todo permanecía conforme a la figura emblemática de los poldavos que es en forma de hélice, y satisfactorio para su animal sagrado que es el caracol (traducción de la autora).

\section{Un poco más de poesía}

En el libro de culto Cent mille milliards de poèmes (Queneau, 1961), Raymond Queneau escribe diez sonetos — catorce versos - impresos sobre diez páginas — uno por página y sólo sobre una de las caras- que se recortan en catorce tiras ${ }^{17}$, cada uno correspondiente a un verso. De este modo ${ }^{18}$, se puede elegir qué soneto leer jugando con estas tiras: por ejemplo, el primer verso del séptimo poema, seguido del segundo verso del décimo, del tercero del primero, etc. ¿Cuántos sonetos pueden leerse siguiendo este sistema? Son cien mil millardos, como reza el título: hay diez elecciones para el primer verso, diez para el segundo y así hasta el verso número catorce, son por lo tanto $10^{14}=100.000 \times 10^{9}$ elecciones posibles - e independientes las unas de las otras-. Queneau explica al principio del libro

\footnotetext{
${ }^{13}$ Véase el Teorema de Bringer, Roubaud y Dumas (Audin, 2010).

${ }^{14}$ Es decir, sólo divisible por sí mismo y por 1.

152 es de orden m módulo $2 \mathrm{n}+1$ si $2^{\mathrm{m}}-1$ es divisible por $2 \mathrm{n}+1$, pero $2^{\mathrm{k}}-1$ no es divisible por $2 \mathrm{n}+1$, para $\mathrm{k}$ menor que $\mathrm{m}$.

${ }^{16}$ Hace referencia al trovador Arnaut Daniel.

${ }^{17}$ Queneau tuvo la idea de escribir este libro observando el álbum para niños Têtes folles, una obra encuadernada en espiral con treinta y dos diseños de otros tantos personajes cortados en cabeza, tronco y piernas; estas tiras horizontales pueden combinarse para obtener figuras humanas insólitas.

18 Queneau escribe al final del prólogo: «Como dijo Lautréamont, la poesía debe estar hecha por todos, no sólo por uno».
} 
cómo los diez sonetos iniciales poseen la misma estructura gramatical, lo que permite que las posteriores elecciones tengan sentido. Además calcula el tiempo necesario para leerlos todos: cuarenta y cinco segundos para leer un poema, quince segundos para cambiar las tiras, ocho horas de lectura al día, doscientos días de lectura al año, es decir, un millón de siglos de lectura.

Como homenaje en el cincuenta aniversario de la publicación de este libro de Queneau, se ha publicado recientemente Cien mil millones de poemas (AA. VV., 2012). Jordi Doce ha sido el creador del modelo de rima - un soneto en alejandrinos de catorce sílabas con cesura en medio, cada verso dividido en dos hemistiquios de siete sílabas-, y todas y todos los demás sonetistas ${ }^{19}$ han respetado ese modelo de rima para crear los $10^{14}$ poemas del mismo modo que en el texto original ${ }^{20}$.

Este bello poema binario (Roubaud, 1995) expresa de manera muy gráfica lo que es el transcurrir de la vida:

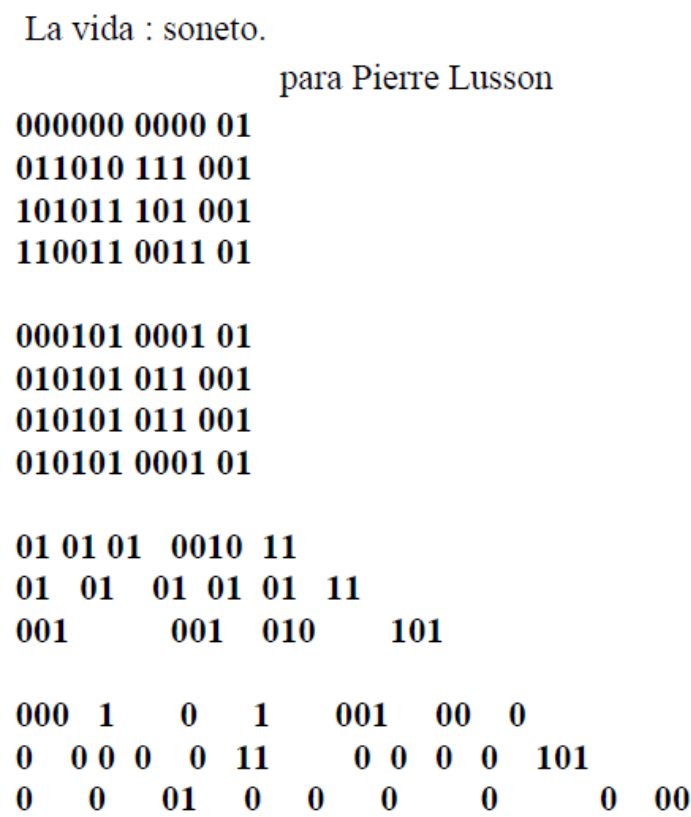

Trente un au cube (Roubaud, 1973) es un poemario de amor de Jacques Roubaud; consta de treinta y un poemas ${ }^{21}$ de treinta y un versos, con treinta y un sílabas cada uno de ellos, por lo que es necesario desplegar las páginas del libro para la lectura. Además, los versos y las sílabas están separados mediante espacios en blanco del modo 5-7-5-7-7=31, sin respetarse la estructura sintáctica - pueden separarse, por ejemplo, preposición y nombre (de / sílabas), nombre y complemento (mallas / del ruido), artículo y sustantivo (las /nebulosas), etc.- .

En su recién publicada Ode à la ligne 29 des autobús parisiens (Roubaud, 2012), Roubaud escribe su oda en alejandrino rimado, siguiendo una serie de reglas que aclara al final del libro. En seis

\footnotetext{
${ }^{19}$ Rafael Reig, Fernando Aramburu, Francisco Javier Irazoki, Santiago Auserón, Pilar Adón, Javier Azpeitia, Marta Agudo, Julieta Valero y Vicente Molina Foix.

${ }^{20}$ El título - Cien mil millones $\left(10^{11}\right)$ de poemas - aparece explicado en el prólogo. En realidad son más, son $10^{15}$ poemas - mil billones de poemas - , porque catorce tiras en blanco esperan al final del libro para que otro soneto —el último, el del lector o lectora- asome, aumentando aún más el tiempo de lectura.

${ }^{21}$ Treinta y uno es el número de sílabas del tanka, un tipo de poesía tradicional japonesa.
} 
cantos de seis estrofas cada uno de ellos —excepto el último que sólo tiene cinco-, el autor va recorriendo las paradas de esa línea de autobús. Con una complicada tipografía, va añadiendo comentarios, aclaraciones, incisos y divagaciones varias con la herramienta del retroceso en la escritura y el cambio de color en el texto.

Una bola de nieve de longitud $n$ es un poema cuyo primer verso está formado por una palabra de una única letra, el segundo de una palabra con dos letras, etc. hasta el $n$-ésimo que consta de un verso con $n$ letras. La siguiente es una bola de nieve - musical— de longitud 21 de Mario Lavista ${ }^{22}$ :

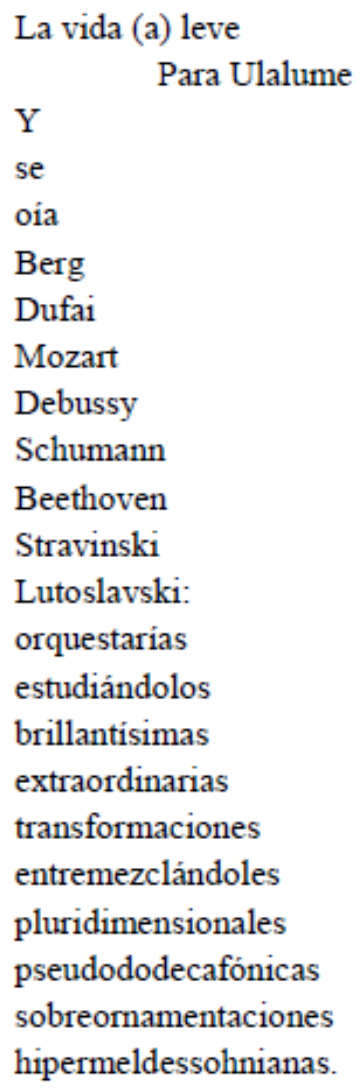

Una bola de nieve de longitud $n$ derritiéndose empieza con un verso de $n$ letras, que se «va derritiendo» hasta llegar a una única letra.

Un rombo es la concatenación de una bola de nieve y una bola de nieve derritiéndose. Liminal Poem para Martin Gardner (Oulipo, 1981) es un conocido rombo de Harry Mathews dedicado al famoso divulgador:

\footnotetext{
${ }^{22}$ http://www.letraslibres.com/blogs/blog-de-la-redaccion/bolas-de-nieve
} 
138 Tropelías. Revista de Teoría de la Literatura y Literatura Comparada, 25 (2016)

Marta Macho Stadler

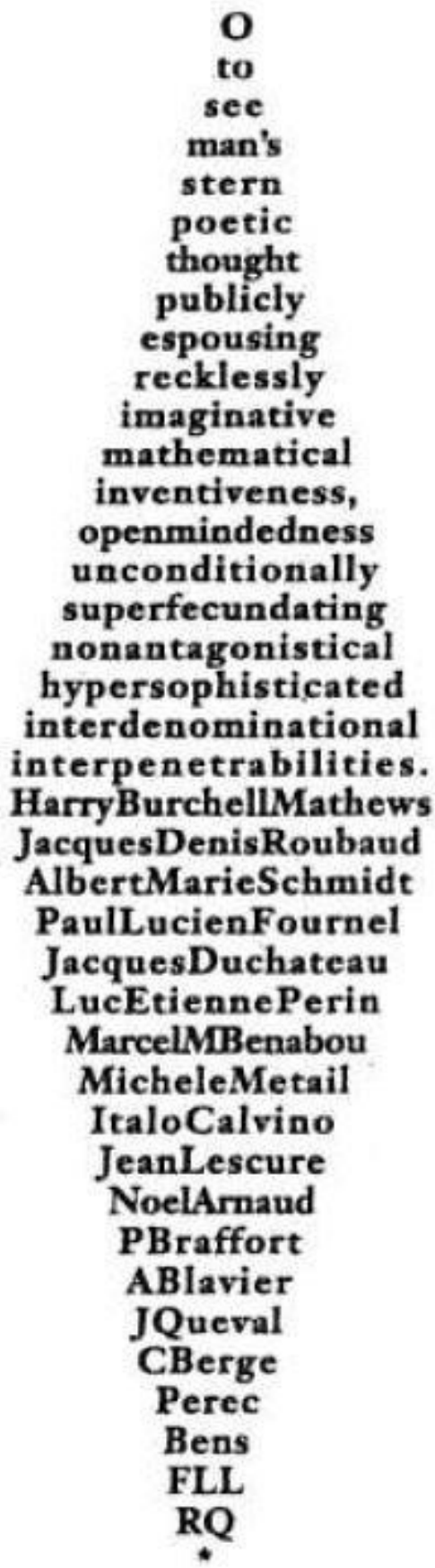

\section{Ejercicios de estilo}

En sus Ejercicios de estilo (Queneau, 2000) ${ }^{23}$, Queneau cuenta una historia anodina de noventa y nueve maneras diferentes. La versión geométrica es una de las divertidas formas matemáticas de narrar la historia:

\footnotetext{
${ }^{23}$ La primera edición de esta obra aparece en 1943 en la revista Messages dirigida por Jean Lescure. Revisada y completada, Gallimard la publica en 1947, y realiza una segunda edición en 1963 acompañada de cuarenta y cinco «exercices de style parallèles peints, dessinés ou sculptés par Jacques Carelman» y de noventa y nueve «exercices de style typographiques» de Robert Massin.
} 
En el paralelepípedo rectangular que se desplaza a lo largo de una línea recta de ecuación $\mathbf{8 4 x}+\mathbf{S}=\mathbf{y}$, un homoide A que presenta un casquete esférico rodeado por dos sinusoides, sobre una parte cilíndrica de longitud $\mathbf{1}>\mathbf{n}$, presenta un punto de intersección con un homoide trivial B. Demostrar que este punto de intersección es un punto de inflexión.

Una de las muchas versiones de este libro — esta idea- se debe a Hervé Le Tellier, que en su Joconde jusqu'à cent: $99(+1)$ points de vue sur Mona Lisa (Le Tellier, 1998) ${ }^{24}$ da diferentes visiones - en clave de humor - de la enigmática protagonista del cuadro de da Vinci. El siguiente es el punto de vista del matemático booleano:

Sea $\boldsymbol{B}$ el conjunto de las mujeres morenas que llevan una redecilla de seda negra en la cabeza, sea $\boldsymbol{I}$ el conjunto de las personas que tienen una sonrisa indefinible, sea $\boldsymbol{P}$ el conjunto de los cuadros de un artista dado $p$.

Probar que si la intersección de $\boldsymbol{I}, \boldsymbol{B}$ y $\boldsymbol{P}$ es La Gioconda, entonces $\boldsymbol{p}$ es Leonardo da Vinci (traducción de la autora).

En Rationnel mon Q. 65 exercices de style (Duchêne-Leblanc, 2010), las autoras demuestran de sesenta y cinco maneras la irracionalidad de la raíz de 2, realizando continuos guiños a textos y a trabas del OuLiPo ${ }^{25}$. Como muestra, el ejercicio de estilo Esperando a $G$ :

-¿Qué hacemos ahora?

- Mientras esperamos.

- Mientras esperamos.

- ¿Y si hiciéramos nuestros ejercicios?

- Nuestros encadenamientos.

- Lógicamente.

- Con aplicación.

- Circunvolución.

- Aplicación.

- Para entrar en calor.

- Para calmarnos.

- Vamos.

Silencio

No se mueven.

(Traducción de la autora.)

Matt Madden (Madden, 2005) tiene una versión en formato de tebeo: Matt está trabajando en su ordenador cuando se levanta para ir a buscar algo a la nevera. Su esposa Jessica le pregunta la hora desde el piso de arriba. Cuando llega a la nevera, Matt no recuerda qué había ido a buscar. Esto se narra, de manera gráfica, de noventa y nueve maneras distintas.

\footnotetext{
${ }^{24}$ Este libro tiene una segunda parte, con más ejercicios de estilo, publicados en 2002.

${ }^{25}$ Aluden a obras de otros autores, como Esperando a Godot de Samuel Beckett, Al otro lado del espejo de Lewis Carroll, Bouvard et Pécuchet de Gustave Flaubert, Je me souviens de Georges Perec, etc. Incluso se hacen referencias al cine - Con la muerte en los talones de Alfred Hitchcock o Barry Lyndon de Stanley Kubrick- y a la pintura de René Magritte.
} 


\section{6.- Geometría y topología marcando el rumbo}

Mai quai Conti (Audin, 2011) es — como su autora Michèle Audin dice en el prefacio- un homenaje a la Comuna de París $^{26}$ en el que se mezclan ciencia, historia y literatura:

i. ciencia porque los trece capítulos — sin contar el prefacio y el epílogocorresponden a trece fechas de 1871, que coinciden con trece sesiones de l'Académie des sciences ${ }^{27}$ que tenían lugar los lunes por la tarde,

ii. historia porque trata de un momento crucial en la historia del pueblo francés: los sesenta días de gobierno de la Comuna, detallándose lo que sucedió en el terreno revolucionario, político y cultural en París, y

iii. literatura porque —además de las muchas referencias literarias que pueden leerse- la autora escribe este texto bajo trabas oulipianas -usa pastiches, tautogramas, monovocalismos, lipogramas, etc.-, y presentando una restricción creada por ella misma, la traba de Pascal, que le permite organizar los capítulos.

En Mai Quai Conti las relaciones entre los personajes están dictadas por las posiciones de los puntos en una figura determinada por el teorema de Pascal $^{28}$.

Si un hexágono está inscrito en una cónica, entonces los tres puntos comunes a los tres pares de lados opuestos están en línea recta.

En el texto, los vértices del hexágono inscrito en la elipse corresponden a Charles Hermite, Joseph Bertrand, Michel Chasles, Charles Delaunay, Léonce Élie de Beaumont y Hervé Faye. Simon Newcomb aparece — en el interior de la elipse — relacionado con algunos de estos personajes; en cada capítulo se van incorporando puntos y personajes, es decir, matemáticas e historia, con el teorema de Pascal como leitmotiv.

\footnotetext{
${ }^{26}$ La Comuna de París fue un movimiento insurreccional que gobernó esta ciudad entre el 18 de marzo y el 28 de mayo de 1871, instaurando un proyecto político popular autogestionario. Regentó París durante sesenta días promulgando una serie de decretos revolucionarios - la autogestión de las fábricas abandonadas por sus dueños, la creación de guarderías para los hijos de las obreras, la laicidad del Estado, la remisión de los alquileres impagados, la abolición de los intereses de las deudas, etc.- - que en su mayoría respondían a la necesidad de paliar la pobreza generalizada que había causado la guerra. La Comuna fue reprimida con extrema dureza: tras un mes de combates, el asalto final al casco urbano provocó una dura lucha en la calle - la Semana Sangrienta - del 21 al 28 de mayo; el balance final fue de unos treinta mil muertos y el sometimiento de París a la ley marcial durante cinco años.

${ }^{27}$ La Academia de Ciencias de Francia se creó en 1666, durante el reinado de Luis XIV, y contó inicialmente con científicos como René Descartes, Blaise Pascal y Pierre de Fermat.

${ }^{28}$ El teorema de Pascal —o Hexagrammum Mysticum Theorem- es un teorema de geometría proyectiva que generaliza el Teorema del hexágono de Pappus y es el dual proyectivo del Teorema de Brianchon. Fue descubierto por Blaise Pascal en 1639.
} 
Usando la clásica banda de Möbius ${ }^{29}$ y gracias a simples manipulaciones, Luc Étienne propone realizar transformaciones sobre un poema que producen un sorprendente cambio de sentido. Se transcribe a continuación el primer método, que el autor denomina lectura directa ${ }^{30}$ :

Se toma una tira de papel muy larga.

Sobre una de sus caras, se escribe la primera mitad del poema ${ }^{31}$.

* Trabajar, trabajar sin cesar,

$\rightarrow$ para mi es obligación

$\rightarrow$ no puedo flaquear

$\rightarrow$ pues amo mi profesión...

Se da la vuelta a la tira a lo largo de su lado más largo, y sobre la segunda cara se escribe la segunda mitad del poema.

Es realmente un tostón

perder el tiempo,

y grande es mi sufrimiento,

cuando estoy de vacación.

Tras realizar un giro de ciento ochenta grados, se pegan las dos extremidades - las cortas - de la tira.

Se obtiene así una banda de Möbius que se lee desde el principio hasta el final sin tener necesidad de dar la vuelta, ya que sólo tiene un lado (el principio está indicado aquí por un asterisco. Al final de cada línea, seguir la flecha).

Trabajar, trabajar sin cesar, es realmente un tostón

para mi es obligación perder el tiempo

no puedo flaquear y grande es mi sufrimiento,

pues amo mi profesión... cuando estoy de vacación.

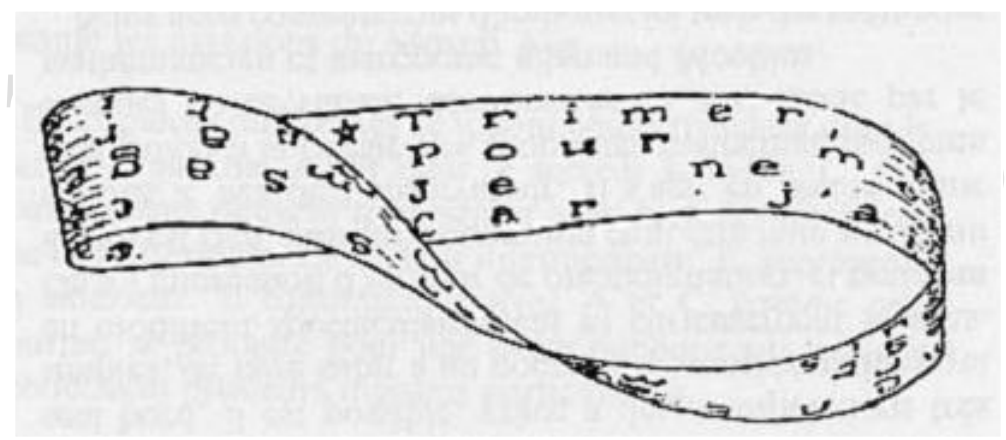

(Oulipo, 1973; traducción de la autora)

Este método ha transformado una oda al trabajo en dos caras en un poema dedicado a la pereza en una única cara. ¡Han sido las propiedades topológicas de la banda de Möbius quienes lo han conseguido!

\footnotetext{
${ }^{29}$ La banda de Möbius es una superficie — tiene dimensión dos- con un único borde y una única cara. La singularidad de este objeto es que no es orientable: intuitivamente, cualquier objeto que viva en una banda de Möbius cambia su orientación al deslizar sobre ella - por ejemplo, una flecha que se mueva sobre esta superficie a lo largo de un circuito cerrado habrá cambiado su sentido al llegar al punto de partida-.

${ }^{30}$ Luc Étienne, Poèmes à métamorphoses pour rubans de Moebius.

${ }^{31}$ Después se explica la razón del asterisco y las flechas.
} 


\section{7. ¿Vamos al teatro?}

En L'arbre à thèâtre (Oulipo, 1973) $)^{32}$ Pierre Fournel propone una comedia con estructura de árbol $^{33}$. Para ello, elabora el grafo que se muestra debajo, ya que - como indica el autor en la introducción del texto- un árbol exigiría un esfuerzo «sobrehumano» de memorización a los actores.

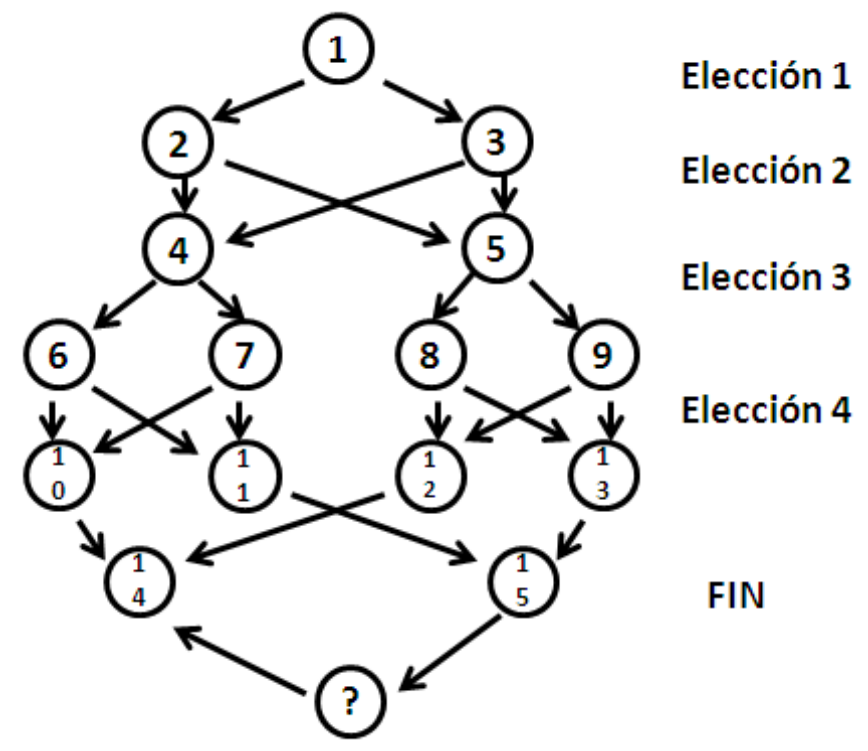

Tomando como base el anterior grafo, los actores interpretan la primera escena y después invitan al espectador a elegir el siguiente cuadro entre los dos posibles -el 2 o el 3-: esta elección puede hacerse a mano alzada — si se está en una sala—, o llamando por teléfono — si la obra se representa en el marco de una emisión radiofónica-; lo esencial es que la votación sea rápida. En la obra que se propone, el espectador deberá elegir cuatro veces, y asistirá a una representación en cinco escenas. Como el grafo propuesto consta de quince escenas - cuatro de las cuales no involucran la elección del espectador - es posible representar dieciséis obras en cinco escenas diferentes. El texto propuesto por Fournel habla de reyes, reinas, princesas, héroes, traiciones, secuestros y duelos, con lo que la diversión está servida.

El Aumento (Perec, 2009) ${ }^{34}$ es una obra teatral de Perec sin acción dramática, con apenas un escenario que debe imaginar el espectador. Los actores ${ }^{35}$ — que no personajes— son: 1. la proposición; 2. la alternativa; 3. la hipótesis positiva; 4. la hipótesis negativa; 5. la elección; 6. la conclusión y la rubeola.

He aquí un fragmento de lo que se puede ver en la obra ${ }^{36}$ :

\footnotetext{
${ }^{32}$ Paul Fournel (con la colaboración de Pierre Énard), L'arbre à théâtre. Comédie combinatoire.

${ }^{33}$ Un árbol es un grafo — conjunto de vértices y aristas uniendo algunos de los anteriores - conexo — de una pieza- y sin ciclos. El grafo propuesto por Fournel es dirigido, es decir, la relación entre los vértices está ordenada.

${ }^{34} \mathrm{La}$ obra lleva como subtítulo o, cómo, sean cuales fueren las condiciones sanitarias, psicológicas, climáticas, económicas o de otra índole, poner de su lado el máximo de oportunidades cuando usted le pide a su jefe de servicio un reajuste de su salario.

${ }^{35}$ Esta nomenclatura pertenece al ámbito de la lógica.

${ }^{36}$ La obra se compone de párrafos similares, repetitivos, obsesivos. Los números son importantes, pues corresponden a cada uno de los actores.
} 
1. Has reflexionado maduramente, has tomado tu decisión y vas a ir a ver a tu Jefe de Servicio para pedirle un aumento de sueldo.

2. O bien tu Jefe de Servicio está en su despacho o no.

3. Si tu Jefe de Servicio estuviera en su despacho, tocarías a la puerta y esperarías su respuesta.

4. Si tu Jefe de Servicio no estuviera en su despacho, esperarías su vuelta en el pasillo.

5. Supongamos que tu Jefe de Servicio no está en su despacho.

6. En este caso, esperas en el pasillo...

Perec juega con la noción de «aumento» en el sentido financiero - el sueldo-, el retórico - apilar una serie de argumentos para llegar a una consecuencia- o matemático.

La obra es una pesadilla sin fin, en la que una multitud de factores entran en juego — si el Jefe de Servicio está en su despacho, si su secretaria Mme. Yolande está de buen o de mal humor, si el Jefe de Servicio ha pasado la cuarentena tras enfermar su hija de rubeola, etc.- construyendo un obsesionante texto combinatorio.

\section{8.- Un libro extraordinario con elaboradas trabas}

En La vida instrucciones de uso (Perec, 1992), Georges Perec relata las historias que suceden en cada uno de los espacios de un edificio imaginario - situado en el número 11 de la calle Simon Crubellier en París - representado en un cuadrado $10 \times 10$ y en una fecha determinada - el 23 de junio de 1975, aproximadamente a las ocho de la tarde-.

En los noventa y nueve capítulos del libro recorremos sótanos, apartamentos, desvanes, tramos de escalera... vidas, manías y personalidades de los inquilinos del edificio, de sus ascendientes, de sus amigos, de sus parientes, etc. El personaje principal — con el que todos están relacionados de alguna manera- es Perceval Bartlebooth, que pasa sus días haciendo y deshaciendo rompecabezas. La obra finaliza con la muerte del protagonista tras realizar un amargo descubrimiento:

Es el veintitrés de junio de mil novecientos setenta y cinco y van a dar las ocho de la tarde. Sentado delante de su puzzle, Bartlebooth acaba de morir. Sobre el paño negro de la mesa, en algún punto del cielo crepuscular del puzzle cuatrocientos treinta y nueve, el hueco negro de la única pieza no colocada aún dibuja la figura casi perfecta de una $\mathbf{X}$. Pero la pieza que tiene el muerto entre los dedos tiene la forma, previsible desde hacía tiempo en su ironía misma, de una $\mathbf{W}$.

Cada capítulo se sitúa en un espacio diferente, con inquilinos distintos, aludiendo a veces a otros habitantes del edificio. Parte del libro se dedica a describir el propio inmueble, y durante este recorrido se dan descripciones ricas en detalles que insisten en materias, colores, formas, estilos, cuadros, etc.

Mil cuatrocientos sesenta y siete personajes desfilan por esta novela, escrita durante los años 1976 a 1978 - aunque Perec llevaba mucho tiempo antes planificándola-, año en la que fue publicada. En Especies de espacios (Perec, 2001) ${ }^{37}$ — publicada en 1974-, Perec habla de lo que en aquel momento era aún un proyecto:

La novela —cuyo título es La Vida instrucciones de uso- se limita (si puedo emplear este verbo para un proyecto cuyo desarrollo final alcanzará algo así como cuatrocientas páginas) a describir las habitaciones

\footnotetext{
${ }^{37}$ Capítulo El inmueble, 1. Proyecto de novela.
} 
puestas al descubierto y las actividades que en ellas se desarrollan, todo ello según procesos formales en cuyo detalle no me parece obligado entrar aquí, pero cuyos solos enunciados me parece que tienen algo de seductor: poligrafía del caballero (y lo que es más, adaptada a un damero de $10 \times 10$ ), pseudo-quenina de orden 10, bi-cuadrado latino ortogonal de orden 10 (aquel que dijo Euler que no existía, pero que fue descubierto en 1960 por Bose, Parker y Shrikhande).

Esas tres trabas citadas se incorporan sin que el lector probablemente sea consciente:

I. La poligrafía del caballero o el orden de recorrido de los lugares del edificio

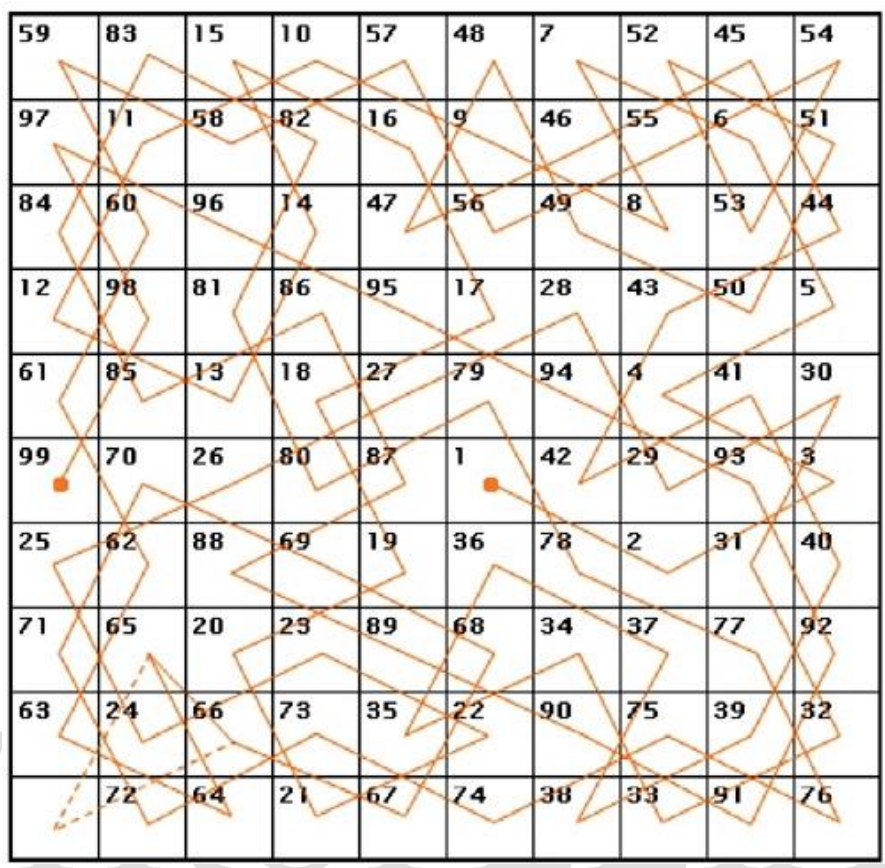

La lectura-recorrido del lector-visitante incorpora una restricción del mundo del ajedrez ${ }^{38}$ : Perec nos obliga a pasar una vez y sólo una por cada lugar del edificio, pero rechaza hacerlo de manera lineal o al azar. Decide usar el modelo de la poligrafía del caballero ${ }^{39}$ que encontró de manera experimental para su tablero-edificio.

Perec se permite una desviación ${ }^{40}$ : en efecto, la casilla del desplazamiento sesenta y seis —que corresponde a un sótano - no se describe y en su lugar se salta al siguiente casilla ${ }^{41}$ que corresponde a la tienda de antigüedades de la señora Marcia:

Y se ha traído del pueblo algunos utensilios y accesorios sin los que no podría pasar: su molinillo de café y su bola para el té, una espumadera, un colador de chino, un pasapuré, un baño maría y la caja en la que, desde siempre ha guardado su vainilla en vainas, su canela en rama, sus clavos de especia, su azafrán, sus granos y su angélica, una vieja caja de galletas de hojalata, cuadrada, en cuya tapa se ve una niña que muerde una punta de una galletita.

La esquina de la galleta corresponde a la casilla inferior izquierda, que tras un mordisco desaparece del juego.

\footnotetext{
${ }^{38} \mathrm{O}$ de la teoría de grafos.

${ }^{39}$ Es un caso particular de grafo hamiltoniano: debe recorrerse todo el tablero pasando una y sólo una vez por cada casilla. En el ajedrez hay sesenta y cuatro casillas, ;pero en el edificio hay cien!

${ }^{40}$ Un clínamen en el lenguaje oulipiano, un cambio local en la traba, es la excepción a la regla.

${ }^{41}$ Por eso el libro tiene noventa y nueve capítulos y no cien.
} 
Una vez fijado el recorrido del edificio, Perec debe llenar cada local descrito, lo que le lleva a dos preguntas: ¿qué poner en cada lugar? ¿dónde poner cada objeto? Procede en dos etapas: elabora veintiún pares de listas de diez elementos a utilizar en cada capítulo-hueco del edificio e idea un algoritmo para distribuir estos elementos de manera no aleatoria.

II. El bicuadrado latino ortogonal de orden 10 o la forma de distribuir las palabras

El edificio se representa como un cuadrado $10 \times 10$ en el que cada casilla-capítulo tiene asignados dos números formando un cuadrado latino ${ }^{42}$ ortogonal $^{43}$. Usando estos pares de números, Perec llega a un cuaderno de cargas (Perec, 1993), en el cual, para cada capítulo, se describe una lista de veintiún pares de temas — autores, mobiliario, animales, colores, sentimientos, música, adjetivos, etc.- que deben figurar en el capítulo ${ }^{44}$.

III. La pseudo-quenina de orden 10 o la forma de permutar las líneas y columnas del bicuadrado

No existen queninas de orden 10, por ello Perec cambia la permutación propuesta por Raymond Queneau ${ }^{45}$ por otra que denomina pseudo-quenina de orden 10 y que le permite generar de manera no aleatoria bicuadrados latinos diferentes, para evitar que para cada casilla se elijan siempre los términos de la misma lista de los veintiún pares elaborados: por ejemplo, en el capítulo veintitrés que corresponde a la casilla $(4,8)$, aparecen los números $(6,5)$, por lo que debe utilizarse una cita de Verne - sexto autor en la primera lista de autores del cuaderno de cargas - y una de Joyce — quinto autor en la primera lista de autores del cuaderno de cargas-, etc.

En mi opinión, este texto es la obra maestra de la literatura oulipiana.

\section{Bibliografía}

AA. VV. (2012): Cien mil millones de poemas. Madrid, Demipage.

Arellano, C., Munárriz, J., y Rhei, S. (2011): Sextinas. Pasado y presente de una forma poética. Madrid, Hiperión.

Audin, M. (2010): «L'Oulipo et les mathématiques. Une description», en http://www-irma.ustrasbg.fr/ maudin/ExposeRennes.pdf (última consulta, 15-12-2015).

(2011): «Mai quai Conti», en http://oulipo.net/fr/mai-quai-conti (última consulta, 15-12-2015).

BenABou, M. (2001): «Quarante siècles d'Oulipo», Magazine littéraire, 398, pp. 20-23.

DuChÈNe, L., y LEBLANC, A. (2010): Rationnel mon Q. 65 exercices de style. Paris, Hermann.

LE Tellier, H. (1998): Joconde jusqu'à cent : $99(+1)$ points de vue sur Mona Lisa. Paris, Castor Astral.

\footnotetext{
${ }^{42}$ Cada dígito está presente una sola vez en cada línea y en cada columna.

${ }^{43}$ Los dos números en la misma casilla sólo se emparejan una vez en ese orden.

${ }^{44}$ A cada par $(a, b)$ del bicuadrado latino le corresponde el elemento $a$ de la primera lista y el $b$ de la segunda. Perec hace aparecer en cada capítulo los cuarenta y dos términos así obtenidos, aunque elabora una estrategia para no realizar esta asignación de manera tan rígida.

${ }^{45}$ La que hemos comentado antes en el apartado 3.
} 
- (2002): Joconde sur votre indulgence. Paris, Castor Astral.

Madden, M. (2005): Exercises in Style (99 Ways to Tell a Story). London, Penguin Books.

OULIPO (1973): La littérature potentielle. Paris, Gallimard.

_ (1981): Atlas de littérature potentielle. Paris, Gallimard.

__ (1987a): La bibliothèque oulipienne I. Paris, Ramsay.

_ (1987b): La bibliothèque oulipienne II. Paris, Ramsay.

_ (2003): La bibliothèque oulipienne VI. Paris, Le Castor Astral.

Perec, G. (1976): Alphabets, Cent soixante-seize onzains hétérogrammatiques. Paris, Galilée.

__ (1992): La vida instrucciones de uso. Trad. Josep Escuer. Barcelona, Anagrama.

_ (1993): Le cahier des charges de la Vie mode d'emploi. Paris, C. N. R. S. et Zulma.

- (2001): Especies de espacios. Trad. Jesús Camarero. Barcelona, Montesinos.

_ (2009): El aumento. Trad. Pablo Moíño Sánchez. Segovia, La uÑa RoTa.

QuenEAU, R. (1961): Cent mille milliards de poèmes. Paris, Gallimard.

- (1965a): Le Chien à la mandoline. Paris, Gallimard.

- (1965b): Bâtons, chiffres et lettres. Paris, Gallimard.

— (2000): Ejercicios de estilo. Trad. Antonio Fernández Ferrer. Madrid, Cátedra.

RoubAUD, J. (1973): Trente un au cube. Paris, Gallimard.

_ (1987): L'enlèvement d'Hortense. Paris, Ramsay.

_ (1990): L'exil d'Hortense. Paris, Seghers.

- (1995): Poesía, etcétera: puesta a punto. Trad. José Luis del Castillo Jiménez. Madrid, Hiperión.

- (2012): Ode à la ligne 29 des autobús parisiens. Paris, Attila. 